\title{
Concepciones de escritura académica en estudiantes de medicina
}

\author{
Conceptions of academic writing in medical students \\ Martha Leticia Gaeta González \\ Mercedes Zanotto González \\ Gabriela González-Ocampo
}

\begin{abstract}
RESUMEN
Este estudio exploratorio tuvo por objetivo identificar el tipo de concepciones de escritura académica de estudiantes de medicina en sus dimensiones epistémica y reproductiva, las cuales es sabido que en las personas pueden presentarse de manera simultánea en niveles distintos, y analizar la relación entre estas y las variables género, edad, semestre y rendimiento académico. Participaron 359 estudiantes de medicina, quienes respondieron al "Instrumento de concepciones sobre escritura académica". Los resultados reportan un nivel elevado de concepciones de tipo epistémico relacionado con todas las variables. Las mujeres obtuvieron un mayor acuerdo con las concepciones epistémicas que los varones y ambos grupos presentaron concepciones reproductivas de nivel medio. No se encontraron diferencias significativas entre las variables edad, semestre y rendimiento académico respecto de las concepciones epistémicas. En cuanto a la dimensión reproductiva no se observó una diferencia significativa referente a la edad, pero sí la hubo en cuanto a los semestres, la cual presentó un nivel medio en los semestres de $1^{\circ}$ a $3^{\circ}$ y bajo en los de $4^{\circ}$ a $10^{\circ}$. Además, los estudiantes de alto rendimiento académico mostraron un nivel medio en creencias reproductivas.
\end{abstract}

Palabras clave: concepciones del estudiante, escritura académica, estudiantes universitarios.

\section{ABSTRACT}

This exploratory study aimed to identify the type of academic writing conceptions of medical students in their epistemic and reproductive dimensions and to analyze the relationship between both dimensions and the variables gender, age, semester and academic performance. A total of 359 medical students completed the "Conceptions on academic writing survey". Results show a high level of epistemic conceptions related to all the variables. Women indicated greater agreement with epistemic conceptions than men and no significant differences were observed regarding reproductive conceptions. Moreover, no significant differences were found between the variables age and semester. Students with the highest academic average showed a higher average level of reproductive beliefs and no significant differences were found regarding epistemic beliefs.

Keywords: student's conceptions, academic writing, university students. 


\section{INTRODUCCIÓN}

En la actualidad existe un amplio consenso respecto del potencial de la escritura académica como herramienta de aprendizaje en los contextos universitarios. Este aprendizaje implica la construcción de conocimientos acerca de un determinado campo disciplinar, así también sobre el propio proceso de escritura académica (utilización del lenguaje escrito en función del género textual, de los objetivos del escritor y de la audiencia) y en lo referente a la autorregulación del pensamiento durante la composición escrita. En este sentido, dado su potencial para el desarrollo de los procesos de pensamiento, la escritura académica ha sido denominada herramienta epistémica (Bereiter y Sacardamalia, 1987, 1992; Miras, 2000; Olson, 1998).

Desde la década de los ochentas, a partir de los estudios fenomenográficos de Hounsell (1984) hasta las investigaciones actuales, se ha puesto de manifiesto que en los docentes y estudiantes existen cierto tipo de representaciones o nociones sobre la escritura académica que favorecen o no la utilización de la escritura como recurso para la construcción de conocimiento o herramienta epistémica (Castelló, Iñesta y Monereo, 2009; Flower, Ackerman y Peck, 1990; Nelson, 1990). Por otra parte, ha sido constatado que estas influyen en la calidad de los textos producidos en estudiantes de secundaria (Miras, Solé y Castells, 2013), de bachillerato (Villalón, Mateos y Cuevas, 2015) y de licenciatura (Boscolo, Arfé y Quarisa, 2007; Campbell, Smith y Brooker, 1998; Lavelle y Zuercher, 2001; Martínez-Fernández, Corcelles, Bañales, Castelló y Gutiérrez-Braojos, 2016; White y Bruning, 2005). A estas representaciones se las ha

Martha Leticia Gaeta González. Universidad Popular Autónoma del Estado de Puebla, México. Es doctora en Psicología y Aprendizaje por la Universidad de Zaragoza, España; maestra en Psicología por la Universidad de las Américas, Puebla. Es académica de tiempo completo en la Facultad de Educación de la Universidad Popular Autónoma del Estado de Puebla. Coordina la Red Interuniversitaria de Investigación Educativa, Puebla (REDIIEP). Es miembro del Sistema Nacional de investigadores, Nivel 1; del Consejo Mexicano de Investigación Educativa (COMIE), de la Red Mexicana de Investigadores de la Investigación Educativa (REDMIIE) y de la Red Nacional de Investigadores en Educación y Valores (REDUVAL). Correo electrónico: marthaleticia.gaeta@upaep.mx. ID: https://orcid.org/0000-0003-1710-217X.

Mercedes Zanotto González. Universidad Nacional Autónoma de México. Es doctora en Psicología de la Educación por la Universidad Autónoma de Barcelona (UAB), España; maestra en Psicología Evolutiva y de la Educación por la UAB. Es académica de tiempo completo en la Dirección General de Orientación y Atención Educativa (DGOAE) de la UNAM. Es vocal del Consejo de Asesor de la DGOAE, docente externo del doctorado en Educación de la Universidad Popular Autónoma del Estado de Puebla. Integrante del Seminario Interuniversitario de Investigación en Estrategias de Enseñanza y Aprendizaje (SINTE) de Barcelona, equipo de investigación consolidado. Correo electrónico: mzanotto@unam.mx. ID: https://orcid. org/0000-0001-5261-2939.

Gabriela González-Ocampo. Universidad Popular Autónoma del Estado de Puebla, México. Es doctora en Educación por la Universitat Ramon Llull de Barcelona, España. Académica de tiempo completo en la Dirección de Innovación Educativa y Desarrollo Académico (DIEDA) de la Universidad Popular Autónoma del Estado de Puebla (UPAEP). Es miembro del Sistema Nacional de investigadores (nivel candidato). Integrante del Grupo de investigación en estrategias de lectura y escritura académica SINTE-Lest. Correo electrónico: gabriela.gonzalez@upaep.mx. ID: https://orcid.org/ 0000-0003-3623-9841. 
denominado "concepciones de escritura" o "creencias sobre escritura"; por nuestra parte utilizaremos dichos términos de manera indistinta.

Las concepciones de escritura, de acuerdo con Castelló, Iñesta y Monereo (2009), corresponden a un amplio rango de representaciones mentales con respecto a los procesos de composición escrita que son más o menos accesibles a la conciencia del estudiante y del docente. Al respecto se han llevado a cabo estudios sobresalientes desde perspectivas teóricas y metodológicas diversas (ver tabla 1). Una de estas corresponde a la fenomenográfica, que concentra el mayor número de estudios y se caracteriza, a grandes rasgos, por enfocarse en un análisis acerca de la manera en que los estudiantes conceptualizan a la escritura académica y se aproximan a esta. Así también se analiza lo que para ellos implica su propio proceso de escritura, todo lo anterior relacionado con el análisis de sus producciones escritas (Boscolo, Arfé

Tabla 1. Enfoques de investigación sobre concepciones de escritura académica.

\begin{tabular}{|c|c|c|}
\hline Perspectivas teórico metodológicas & Estudios relevantes & Hallazgos generales en investigación \\
\hline $\begin{array}{l}\text { Estudios fenomenográficos } \\
\text { (mayoritarios). Estudian: discurso } \\
\text { sobre elaboración de textos y tipos } \\
\text { de textos, aproximaciones a la } \\
\text { escritura, metáforas sobre el proceso } \\
\text { de escritura } \\
\text { Fundamentados en Märton y Säljö } \\
(1976)\end{array}$ & $\begin{array}{l}\text { (Hounsell, 1984; } \\
\text { Campbell, Smith } \\
\text { y Brooker, 1998; } \\
\text { Lavelle y Zuercher, } \\
\text { 2001; Boscolo, Arfé } \\
\text { y Quarisa, 2007; } \\
\text { Levin y Wagner, } \\
\text { 2006) }\end{array}$ & $\begin{array}{l}\text { Concepción simple: enfocada a la acumulación de información, } \\
\text { acordes con el modelo de "decir el conocimiento", escritos de tipo } \\
\text { reproductivo. Perspectiva transmisiva del aprendizaje y asociada a } \\
\text { textos de calidad baja. } \\
\text { Concepción constructiva: modelo de "transformar el conocimiento"; } \\
\text { desarrollo de argumentos, integración estructurada de contenidos. } \\
\text { Reflexiva y elaboración de ideas. Enfoque profundo del aprendizaje } \\
\text { y calidad alta de los textos escritos. }\end{array}$ \\
\hline $\begin{array}{l}\text { Estudios que integran aspectos } \\
\text { cognitivos, epistémicos, emocionales } \\
\text { y motivacionales. Estudian } \\
\text { problemas de escritura e ideas } \\
\text { adaptativas. }\end{array}$ & $\begin{array}{l}\text { (Lonka et al., 2014; } \\
\text { Martínez-Fernández } \\
\text { et al., 2016) }\end{array}$ & $\begin{array}{l}\text { Sentimientos de ansiedad, estrés, exhaustividad y falta de } \\
\text { interés correlacionaron positivamente con problemas en la } \\
\text { escritura científica (procrastinación, bloqueo, perfeccionismo) y } \\
\text { correlacionaron negativamente con productividad. } \\
\text { La perspectiva de escritura vinculada con la transformación del } \\
\text { conocimiento se relacionó positivamente con la productividad auto- } \\
\text { reportada y se relacionó negativamente con creer que la escritura } \\
\text { es una habilidad innata. }\end{array}$ \\
\hline $\begin{array}{l}\text { Estudios sobre teorías implícitas } \\
\text { en escritura }\end{array}$ & $\begin{array}{l}\text { White y Bruning } \\
(2005)\end{array}$ & $\begin{array}{l}\text { Creencias transmisivas: la escritura consiste en transferir información } \\
\text { de fuentes autorizadas al lector a partir del texto elaborado. }\end{array}$ \\
\hline $\begin{array}{l}\text { Estudios sobre teorías implícitas } \\
\text { de escritura en distintas disciplinas }\end{array}$ & $\begin{array}{l}\text { (Hernández, 2012, } \\
\text { 2017; Hernández y } \\
\text { Rodríguez, 2018) }\end{array}$ & $\begin{array}{l}\text { Creencias transaccionales: la escritura como una construcción } \\
\text { personal y analítica de las fuentes consultadas mediante la integración } \\
\text { activa de su propio pensamiento en el proceso. }\end{array}$ \\
\hline $\begin{array}{l}\text { Estudios sobre concepciones } \\
\text { implícitas de escritura y } \\
\text { construcción de conocimiento }\end{array}$ & $\begin{array}{l}\text { (Villalón y Mateos, } \\
\text { 2009; Villalón, 2010; } \\
\text { Castells et al., } 2015 \text { y } \\
\text { Villalón, Mateos y } \\
\text { Cuevas, 2015) }\end{array}$ & $\begin{array}{l}\text { Concepción reproductiva: próxima a un modelo de "Decir el } \\
\text { conocimiento", en el que la escritura se utiliza como recurso para } \\
\text { transmitir y reproducir lo que se sabe. } \\
\text { Concepción epistémica: la escritura se utiliza como herramienta } \\
\text { conceptual y retórica, acorde con el modelo de "Transformar el } \\
\text { conocimiento". }\end{array}$ \\
\hline
\end{tabular}


y Quarisa, 2007; Campbell, Smith y Brooker, 1998; Hounsell, 1984; Lavelle, 1993; Lavelle y Zuercher, 2001; Lavelle y Bushrow, 2007; Levin y Wagner, 2006).

Por otra parte, se han realizado investigaciones por Lonka, Chow, Keskinen, Hakkarainen, Sandström y Pyhältö (2014) que abordan las concepciones de escritura de manera integrada con factores de tipo cognitivo y epistémico, fundamentados en los modelos de Bereiter y Scardamalia (1987) y los aportes de Bandura (1982), así también en relación con factores emocionales y motivacionales (Boice, 1993). Otra perspectiva de relevancia en el abordaje de las concepciones de escritura es la que corresponde a las creencias implícitas, en la cual se encuentran los trabajos de White y Bruning (2005). Los autores comprobaron la existencia de dos tipos de creencias implícitas de escritura, una de ellas es transmisiva y la otra transaccional, las cuales a su vez se asocian a aquellas creencias de tipo reproductivo y constructivo o epistémico respectivamente, que han sido abordadas por distintos autores que retoman a este enfoque como uno de sus referentes clave (Castells, Mateos, Martín, Solé y Miras, 2015; Hernández, 2012, 2017; Hernández y Rodríguez, 2018; Villalón, 2010; Villalón y Mateos, 2009).

Las perspectivas mencionadas, en su mayoría, retoman los modelos de escritura que aportaron las investigaciones pioneras de Bereiter y Scardamalia (1987; 1992), las cuales contribuyeron al logro de una mayor comprensión sobre el modelo de escritura que caracteriza a las estrategias de los escritores expertos, designado "transformación del conocimiento" (knowledge-transforming), y el modelo de escritura de los escritores aprendices o de "decir el conocimiento" (knowledge-telling). Asimismo, los modelos de los autores permiten el entendimiento de cómo se favorece o no la construcción de nuevo conocimiento a partir de determinadas representaciones de la escritura que tienen los escritores expertos y aprendices, las cuales a su vez se relacionan con los procesos de composición escrita que llevan a cabo.

El desempeño de los escritores aprendices, que corresponde al modelo denominado "decir el conocimiento", knowledge-telling, se caracteriza por no generar una interacción entre los conocimientos conceptuales y los aspectos retóricos (objetivos o intencionalidad del autor en relación con la representación que tiene de la audiencia del texto) del proceso de escritura. Por lo anterior, no logra dirigirse adecuadamente a la audiencia o supuesto lector. De este modo, el escritor desarrolla el texto con un enfoque orientado únicamente a abordar lo que conoce sobre la temática a tratar en el mismo, lo que no implica una nueva elaboración del contenido a escribir y con ello no se propicia el desarrollo de aprendizajes. Así, por ejemplo, lo narrativo puede resultar demasiado predecible y lo argumentativo poco persuasivo. En el caso de los escritores expertos, su desempeño se caracteriza por la puesta en marcha del modelo "transformar el conocimiento", knowledge-transforming, el cual establece una estrecha relación entre los aspectos de contenido conceptual y los retóricos, lo que permite la obtención de un texto ajustado al lector al que se dirige, además de favorecer en 
el escritor la reelaboración de información y la generación de nuevas ideas en torno al tema desarrollado en el texto.

Otro de los enfoques que retoma los trabajos clásicos de Bereiter y Scardamalia (1987, 1992), así como las creencias implícitas de White y Bruning (2005) y el enfoque de las teorías implícitas de la enseñanza y el aprendizaje (Pozo, Scheuer, Mateos y PérezEcheverría, 2006), es el que comprende a las concepciones de la escritura académica como teorías implícitas en vinculación con procesos de aprendizaje (Villalón, 2010; Villalón y Mateos, 2009). Este enfoque lo hemos considerado como aquel con posibilidades claras para analizar los aspectos epistémicos de las concepciones de escritura. Esta perspectiva a su vez pone de manifiesto la existencia de una relación bidireccional entre las concepciones de escritura y las prácticas de composición escrita. Así, el modo en el que los estudiantes se aproximan y se enfrentan a las tareas o prácticas de escritura para aprender, se encuentra mediado por sus concepciones sobre la escritura, a la vez que las propias prácticas de escritura les requieren procesos o estrategias que pueden influir en el desarrollo de determinadas concepciones sobre la composición escrita.

En el estudio de Villalón y Mateos (2009) realizado con alumnos de secundaria y universitarios, fueron identificadas dos tipos de concepciones vinculadas con los dos modelos de Bereiter y Scardamalia $(1987,1992)$. Una de ellas concierne a una concepción reproductiva, próxima a un modelo de "decir el conocimiento", en el que la escritura se utiliza como un recurso para transmitir y reproducir lo que se sabe. La otra concepción es de tipo epistémico, que corresponde a una comprensión de la escritura como herramienta conceptual y retórica, acorde con el modelo de "transformar el conocimiento". Este tipo de estudio es relevante para la comprensión de dos clases distintas de concepciones sobre la escritura que poseen los estudiantes y entender el tipo de impacto que estas pueden generar en las prácticas escritas, a su vez señala la dificultad que existe para la toma de consciencia de la función epistémica de la escritura por parte de los estudiantes y de la necesidad de propiciar el desarrollo de un trabajo de reflexión metacognitiva que favorezca la resolución de dichas dificultades (Villalón, 2010).

Las concepciones de escritura determinadas por Villalón y Mateos (2009), al igual que las creencias transmisionales y transaccionales comprobadas por White y Bruning (2005), se consideran fundamentadas en supuestos o principios no articulados o implícitos, poco accesibles a la consciencia. Asimismo, dado su carácter implícito, en los estudiantes no se presenta una total consistencia en aquellas reproductivas y epistémicas, es decir, no se observan de manera pura, sino que sus concepciones tienen características de ambas. Acorde con ello, White y Bruning (2005) evidenciaron que estas resultan independientes estadísticamente entre sí, por lo que fue posible que los escritores tuviesen niveles altos y bajos de cada creencia y generaran configuraciones de creencias implícitas de escritura tales como transaccional baja/alta transmisión y transaccional alta/baja transmisión. 
Por su parte, cabe señalar que si bien en México contamos con aportes valiosos en investigación sobre creencias implícitas en escritura académica (Hernández, 2012, 2015; Hernández y Rodríguez, 2018), los estudios son insuficientes y debido a esto el desarrollo de líneas de investigación al respecto es incipiente. Es por ello que resulta necesario obtener un mayor conocimiento respecto de determinadas prácticas de escritura en el contexto universitario y en distintas disciplinas que promueven en los estudiantes un uso epistémico de las mismas y una calidad alta de las producciones escritas, así como también sobre los tipos de prácticas asociadas a concepciones de escritura reproductivas y a una baja calidad de los textos elaborados.

En lo que concierne a los estudios sobre concepciones de escritura en el campo disciplinar de la medicina, estos resultan escasos. Sin embargo, la investigación sobre las representaciones de la escritura académica en estudiantes de medicina es importante, pues en la actualidad cada vez se genera mayor consenso respecto de la necesidad de que dichos alumnos mejoren su capacidad para publicar las investigaciones que realizan, la cual está asociada al desarrollo de aptitudes científicas en este campo del conocimiento (Barbón y Bascó, 2016; Ríos-González, 2016). A su vez, desde la psicología de la educación sabemos que dicha capacidad también se encuentra vinculada con las prácticas de escritura académica que se realizan en un campo disciplinar determinado, las cuales pueden promover en mayor o menor medida concepciones epistémicas y reproductivas que favorezcan o no respectivamente la producción científica por parte de los estudiantes (Hernández, 2012, 2015).

Además de la indagación de las concepciones de escritura en estudiantes de medicina, resulta de interés identificar el tipo de concepciones en relación con el género, dada la relevancia de observar si los puntajes más elevados en concepciones epistémicas de las mujeres, comparado con el de los varones, además de presentarse en el nivel de bachillerato también se observan en el universitario, con una disminución en el puntaje de las concepciones reproductivas (Villalón, Mateos y Cuevas, 2015). Por su parte, distintos estudios que comparan el tipo de concepciones de escritura entre estudiantes de secundaria y universitarios (Villalón y Mateos, 2009), entre alumnos de secundaria, bachillerato y universitarios (Hernández, 2017) y estudiantes de bachillerato y universitarios (Hernández y Rodríguez, 2018) arrojan resultados que indican mayor acuerdo con concepciones epistémicas o constructivas por parte de los universitarios y mayor desacuerdo con las concepciones reproductivas. Aunque no se presenta una disminución significativa de concepciones reproductivas en la mayoría de los casos de los universitarios, comparados con los de secundaria y bachillerato.

Acorde con la importancia de comparar distintos niveles educativos, también sería necesario analizar qué tanto el grado de las creencias epistémicas y reproductivas de escritura guarda relación con la magnitud de experiencia de los estudiantes en el contexto universitario, pues sería plausible que en los semestres más avanzados los estudiantes requiriesen, a diferencia de los semestres iniciales, más habilidades de 
escritura de un alto nivel de desarrollo asociadas a concepciones epistémicas elevadas y a aquellas reproductivas en un bajo nivel (Castells et al., 2015). De la misma manera, en el entendido de que los estudiantes de menor edad generalmente se encuentran en los semestres iniciales y los de mayor edad en los últimos, es de importancia indagar la relación de las edades de los estudiantes universitarios con el nivel de creencias epistémicas y reproductivas.

Otro aspecto de interés es la relación existente entre concepciones de escritura transaccionales o de tipo epistémico y un nivel bajo de concepciones reproductivas con una mayor calidad de la producción escrita en estudiantes universitarios (Boscolo, Arfé y Quarisa, 2007; Campbell, Smith y Brooker, 1998; Hounsell, 1984; Lavelle y Zuercher, 2001; Martínez-Fernández et al., 2016; White y Bruning, 2005) de un campo disciplinar determinado, en caso de que en este se promuevan prácticas de escritura académica enfocadas a la construcción de aprendizajes, lo que podría contribuir a la obtención de un elevado promedio de calificaciones. Sin embargo, sería necesario generar mayor investigación que permita comprender mejor la relación entre concepciones de escritura, tipos de prácticas de escritura y desempeño académico en los campos disciplinares.

Acorde con lo previo, en el presente estudio exploratorio hemos formulado las siguientes preguntas de investigación: ¿Qué tipo de concepciones sobre la escritura académica presentan los estudiantes de la licenciatura en medicina? ¿Existen diferencias en las concepciones de escritura académica en función del género, la edad, el semestre y el rendimiento académico? Conforme a ello, este estudio tiene los siguientes objetivos:

1) Identificar las concepciones de escritura académica de estudiantes universitarios en función de su tipo epistémico y reproductivo.

2) Comparar las concepciones de escritura académica de acuerdo con las variables sociodemográficas: género, edad, semestre y rendimiento académico (considerado para fines de este estudio como el promedio de calificaciones).

Consideramos que esta investigación nos permitirá lograr una mayor comprensión de las representaciones que los estudiantes de medicina tienen acerca de los procesos de composición escrita académica en sus distintas dimensiones epistémicas y reproductivas.

\section{MÉtodo}

Este estudio se desarrolló desde un enfoque cuantitativo, mediante un diseño exploratorio y de asociación, a fin de identificar las concepciones de escritura académica de estudiantes de la licenciatura en medicina en sus dimensiones epistémica y reproductiva, además de analizar la relación entre estas y las variables sociodemográficas: género, edad, semestre y rendimiento académico. 


\section{Participantes}

Participaron en el estudio 359 estudiantes, de $1^{\circ}$ a $10^{\circ}$ semestres, de la Facultad de Medicina de una universidad localizada en la ciudad de Puebla, México. De ellos, 220 son mujeres (61\%) y $139(39 \%)$ son varones, entre 17 y 28 años (media $=19.89 \pm$ 1.95). Los estudiantes tuvieron una participación voluntaria y se garantizó la confidencialidad de la información.

\section{Instrumento}

En el presente estudio se aplicó el "Instrumento de concepciones sobre escritura académica”, diseñado con el propósito de identificar las concepciones de escritura académica de los estudiantes universitarios. El instrumento de 35 ítems toma como fundamento de su diseño a White y Bruning (2005) y a Villalón (2010). Este instrumento de auto-informe tiene una escala de respuesta tipo Likert de cinco opciones que van de 1 (totalmente en desacuerdo) a 5 (totalmente de acuerdo).

$\mathrm{El}$ análisis psicométrico del instrumento mostró la existencia de dos factores con auto-valores superiores a uno, que explicaban el $28 \%$ de la varianza total; el factor que integra las concepciones de tipo epistémico, de 18 ítems, y el factor que integra las concepciones de tipo reproductivo, de 17 ítems (ver tabla 2).

El grado de fiabilidad (alpha de Cronbach) del cuestionario total fue de 0.74, el cual se considera adecuado, así como el de cada uno de los factores; concepciones de tipo epistémico $(\alpha=0.87)$ y concepciones de tipo reproductivo $(\alpha=0.80)$.

\section{Procedimiento y análisis de los datos}

La recolección de datos se llevó a cabo de manera electrónica, después de contar con el consentimiento por parte de las autoridades de la institución sobre los objetivos y el procedimiento del estudio.

Los datos fueron analizados mediante el programa estadístico Statistical Package for the Social Sciences (SPSS) versión 22. En primer lugar se llevaron a cabo análisis descriptivos, a fin de determinar las distintas dimensiones de concepciones de escritura académica por parte de los estudiantes. Posteriormente se efectuó un análisis comparativo respecto de las concepciones de escritura académica por grupo, con respecto al género, a la edad, al semestre académico que cursaban y al rendimiento académico. A continuación se indica la conformación de los distintos grupos.

Respecto de la edad de los estudiantes, se conformaron tres grupos: 17-19 años, 20-22 años, 23-27 años. Así también respecto al semestre, se integraron tres grupos que incluyen a todos los semestres de la carrera de medicina: $1^{\circ}-3^{\circ}, 4^{\circ}-6^{\circ}, 7^{\circ}-10^{\circ}$. En cuanto al rendimiento académico se dividió a los estudiantes en dos grupos: el primero correspondiente a un promedio 7.0-8.9 y el segundo grupo a un promedio 9.0-10. 
Tabla 2. Ítems del intrumento y los factores correspondientes.

Concepción de tipo reproductivo de la escritura

1. Para saber si un texto está bien escrito, siempre hay que tener en cuenta los mismos criterios

2. Si un texto está correctamente escrito, a todo el mundo le resulta útil

3. Me parece que lo único importante en un texto es que aparezcan todas las ideas principales de forma lógica

4. Si quisiera escribir mejor, solo tendría que practicar mucho 5 .

5. Para escribir suelo tomar en cuenta solo mi punto de vista 6 . y no el de quien lo leerá

6. Considero que no hace falta argumentar las ideas de mi escrito si la información que presento es suficientemente clara

7. No suelo pensar en las características principales del tipo de texto que tengo que escribir

8. Si en mi texto presento información amplia y clara no es necesario pensar en si será de interés para quien lo lea

9. Termino un texto cuando se me acaban las ideas, cuando ya no se me ocurre qué más decir

10. Mientras escribo solo pienso en qué ideas tengo que decir

11. Una vez que sé qué ideas tengo que comunicar, me parece que el texto está casi hecho

12. Escribo el texto sin antes tomar notas o realizar esquemas

13. Creo que la organización de las ideas se logra al momento de escribir el texto tal y como quedará al final

14. Considero que una sola versión del escrito es suficiente para tener un texto definitivo que pueda ser entregado

15. Solo releo lo que he escrito cuando considero concluido el texto

16. Al volver a leer lo que he escrito, lo que más corrijo son palabras, signos de puntuación y faltas

17. Cuando releo el texto que escribí es solo para hacer pequeños cambios
Concepción de tipo epistémico de la escritura

1. Escribir me ayuda a observar la complejidad de las ideas

2. Escribir me permite entender mejor lo que estoy pensando

3. Cuando estoy leyendo algo que quiero aprender suelo tomar notas, hacer resúmenes, elaborar esquemas, etc.

4. Plantearme para qué estoy escribiendo y cuál es la mejor forma de conseguirlo me ayuda a escribir mejor

5. Escribir me ayuda a organizar lo que pienso

6. Antes de empezar a escribir pienso en los propósitos que quiero lograr con mi texto

7. Antes de comenzar a escribir trato de que me quede claro el tipo de texto que tendré que redactar

8. Pienso en cómo quiero que los lectores reaccionen frente a mi texto

9. Antes de empezar a escribir pienso en qué características debe presentar el texto dependiendo de quién lo leerá

10. Aunque tenga muchas ideas sobre lo que voy a escribir, creo que todavía tengo que revisarlas y organizarlas hasta que las ponga por escrito

11. A la hora de escribir, pienso en cómo desarrollar mejor mis ideas en función de quién es el lector

12. Mientras escribo tengo en mente mi propósito de escritura

13. Suelo tomar notas y hacer esquemas antes de escribir

14. Cuando tengo que elaborar un texto para una asignatura hago varias versiones antes de llegar al escrito que finalmente entrego

15. Cuando releo mi texto a veces me parece que una idea debería ir en otro lugar

16. Cuando releo lo que estoy escribiendo las ideas a desarrollar en el texto se vuelven más claras

17. Me gusta que alguien más lea mi texto antes de entregarlo para ver si se entiende

18. Cuando releo mi texto, muchas veces se me ocurre una forma mejor de decir una idea

Fuente: adaptado de Villalón (2010), con permiso de la autora.

\section{Resultados}

A continuación se presentan los principales resultados del estudio, con la finalidad de responder a los objetivos de la investigación.

\section{Concepciones de escritura académica por rangos}

Con el propósito de identificar las concepciones de escritura académica que presentan los estudiantes (de tipo epistémico y de tipo reproductivo) establecimos tres rangos 
(bajo, medio y alto), con base en los puntajes mínimo y máximo de las escalas del cuestionario (tabla 3). A partir de ello ubicamos los puntajes medios de los participantes por grupo en cuanto al género, a la edad, al semestre y al rendimiento académico.

Tabla 3. Rangos de las concepciones sobre escritura académica.

Concepción de tipo epistémico

\begin{tabular}{cc}
\hline Rango & Puntaje \\
\hline Bajo & $17.0-39.9$ \\
\hline Medio & $40.0-62.9$ \\
\hline Alto & $63.0-85.0$ \\
\hline
\end{tabular}

Concepción de tipo reproductivo

\begin{tabular}{cc}
\hline Rango & Puntaje \\
\hline Bajo & $18.0-41.9$ \\
\hline Medio & $42.0-65.9$ \\
\hline Alto & $66-0-90.0$ \\
\hline
\end{tabular}

Fuente: construcción personal..

En la tabla 4 se presentan las concepciones de tipo epistémico y las concepciones de tipo reproductivo, de acuerdo con el género. Tanto las mujeres como los varones se encuentran dentro del rango alto de concepciones de tipo epistémico y dentro del rango medio de tipo reproductivo (figura 1).

Tabla 4. Descriptivos de concepciones reproductivas y epistémicas por género.

\begin{tabular}{lcccc} 
Concepciones de escritura académica & Género & $\mathrm{n}$ & Media & Desviación estándar \\
\hline Epistémicas & Mujer & 220 & 76.20 & 7.79 \\
\cline { 2 - 5 } & Hombre & 139 & 73.27 & 8.30 \\
\hline Reproductivas & Mujer & 220 & 42.05 & 8.38 \\
\cline { 2 - 5 } & Hombre & 139 & 42.14 & 8.50 \\
\hline
\end{tabular}

Fuente: construcción personal.

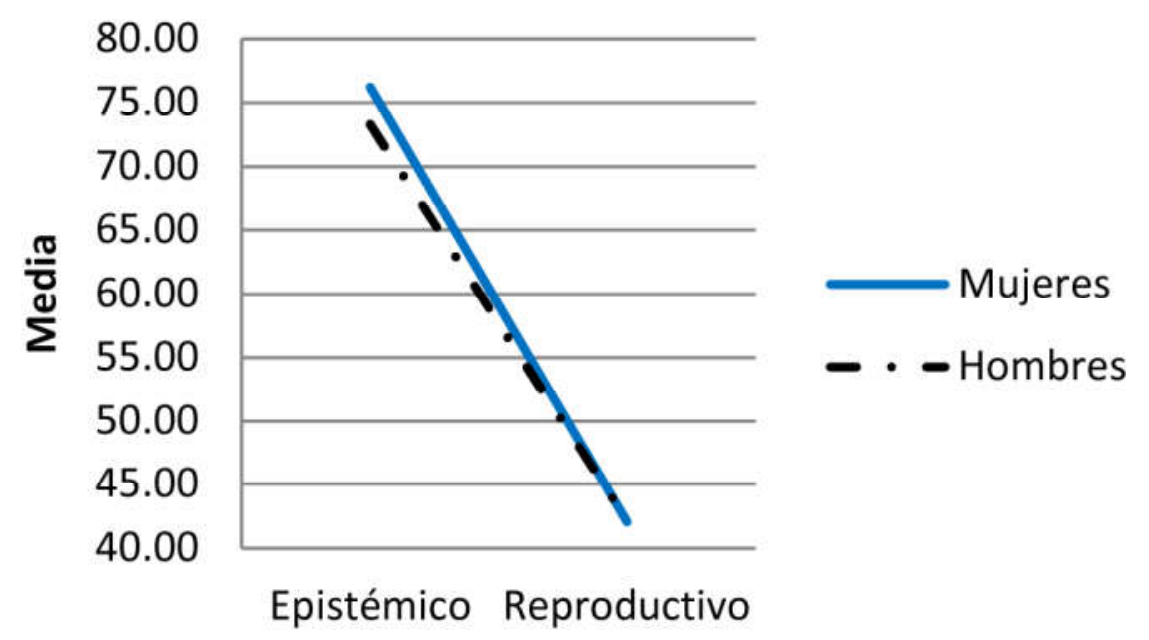

Figura 1. Medias por género respecto a las concepciones de tipo epistémico y de tipo reproductivo.

Fuente: construcción personal. 
En la tabla 5 se muestran las concepciones de tipo epistémico y las concepciones de tipo reproductivo, de acuerdo con la edad. Cabe señalar que dos estudiantes no reportaron su edad, por lo que no fueron considerados en el análisis. Respecto de las concepciones de tipo epistémico, los tres grupos de edad se encuentran dentro del rango alto. En cuanto a las concepciones de tipo reproductivo, los estudiantes con edades 17-19 años se ubican dentro del rango medio, pero los estudiantes con edades 20-22 y 23-27 se encuentran dentro del rango bajo (figura 2).

Tabla 5. Descriptivos de concepciones reproductivas y epistémicas por edad.

\begin{tabular}{lcccc} 
Concepciones de escritura académica & Rango de edad & $\mathrm{n}$ & Media & Desviación estándar \\
\hline Epistémicas & $17-19$ & 200 & 75.39 & 7.18 \\
\cline { 2 - 5 } & $20-22$ & 119 & 74.78 & 75.24 \\
\cline { 2 - 5 } & $23-27$ & 38 & 42.57 & 7.92 \\
\hline & $17-19$ & 200 & 41.48 & 9.22 \\
\cline { 2 - 5 } & $20-22$ & 119 & 41.03 & 9.23 \\
\hline
\end{tabular}

Fuente: construcción personal.

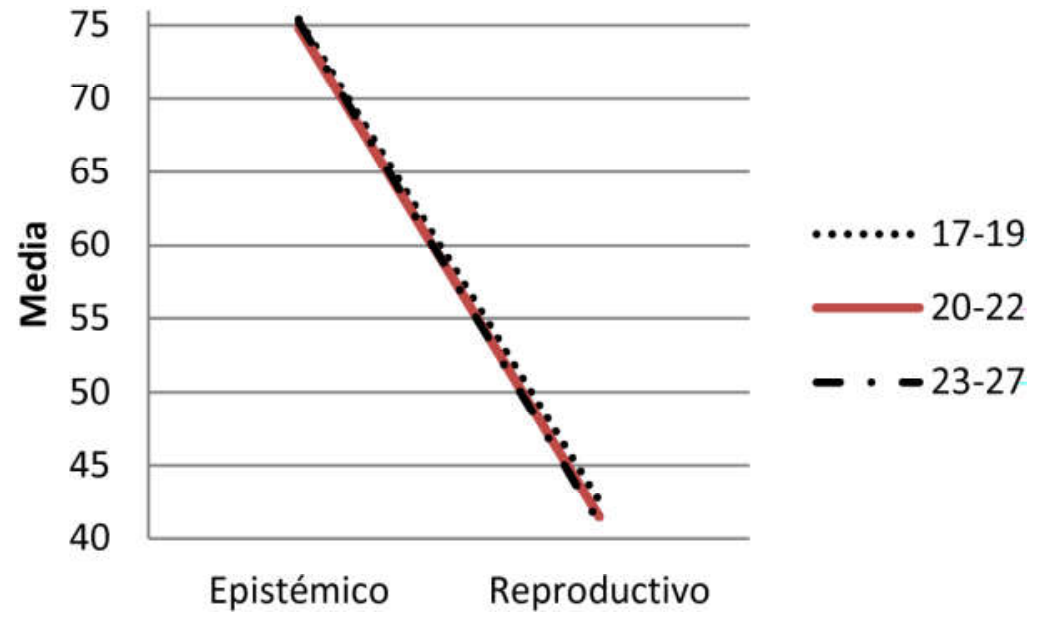

Figura 2. Medias por edad respecto a las concepciones de tipo epistémico y de tipo reproductivo.

Fuente: construcción personal.

En la tabla 6 se muestran las concepciones de tipo epistémico y las concepciones de tipo reproductivo, de acuerdo con el semestre. Respecto a las concepciones de tipo epistémico, los tres grupos se encuentran dentro del rango alto. En cuanto a las concepciones de tipo reproductivo, los estudiantes que cursan los semestres 1-3 se encuentran dentro del rango medio, sin embargo, los estudiantes que cursan 4-6 y 7-10 se encuentran dentro del rango bajo (figura 3). 
Tabla 6. Descriptivos de concepciones reproductivas y epistémicas por semestre.

\begin{tabular}{lcccc} 
Concepciones de escritura académica & Semestre & $\mathrm{n}$ & Media & Desviación estándar \\
\hline Epistémicas & $1-3$ & 231 & 75.01 & 7.50 \\
\cline { 2 - 5 } & $4-6$ & 47 & 75.94 & 7.56 \\
\cline { 2 - 5 } & $7-10$ & 81 & 74.73 & 9.47 \\
\hline Reproductivas & $1-3$ & 231 & 42.81 & 39.87 \\
\cline { 2 - 5 } & $4-6$ & 47 & 41.32 & 9.24 \\
\hline
\end{tabular}

Fuente: construcción personal.

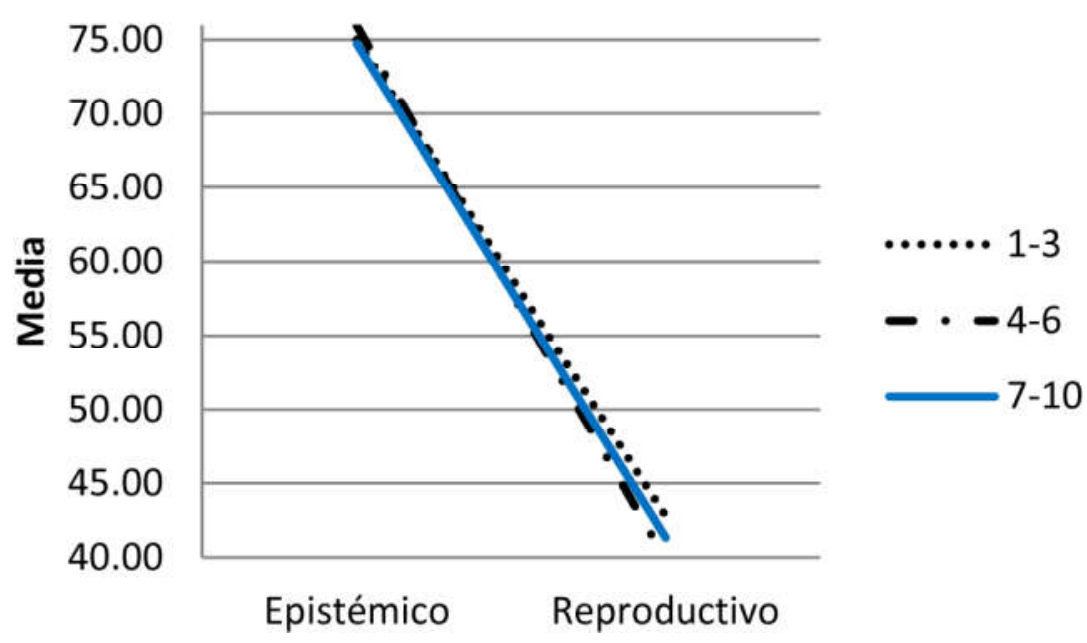

Figura 3. Medias por semestre respecto a las concepciones de tipo epistémico y de tipo reproductivo.

Fuente: construcción personal.

En la tabla 7 se muestran las concepciones de tipo epistémico y las concepciones de tipo reproductivo, de acuerdo con el rendimiento académico. Los dos grupos se encuentran dentro del rango alto de concepciones de tipo epistémico. En lo que respecta a las concepciones de tipo reproductivo, los estudiantes con un rendimiento académico 7-8 se encuentran dentro del rango bajo, sin embargo, los estudiantes con un rendimiento académico 9-10 se ubican dentro del rango medio (figura 4).

Tabla 7. Descriptivos de concepciones reproductivas y epistémicas por rendimiento académico.

\begin{tabular}{lcccc} 
Concepciones de escritura académica & Rendimiento académico & $\mathrm{n}$ & Media & Desviación estándar \\
\hline Epistémicas & $7-8$ & 199 & 75.01 & 8.23 \\
\cline { 2 - 5 } & $9-10$ & 160 & 75.14 & 7.98 \\
\hline Reproductivas & $7-8$ & 199 & 40.75 & 43.75 \\
\hline
\end{tabular}

Fuente: construcción personal. 


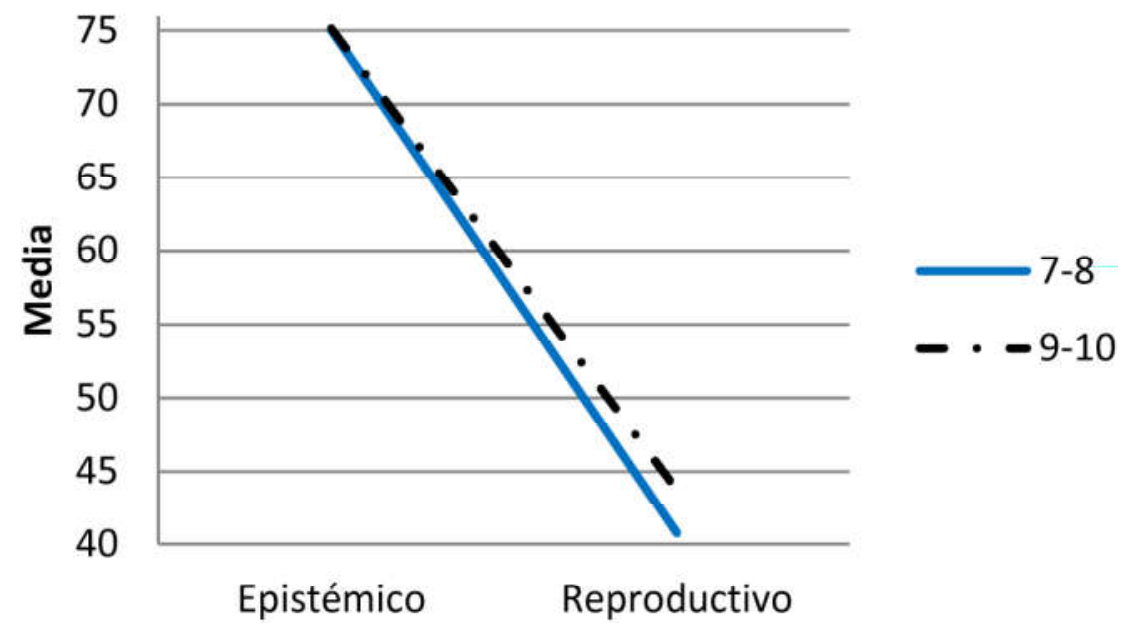

Figura 4. Medias por rendimiento académico respecto de las concepciones de tipo epistémico y de tipo reproductivo.

Fuente: construcción personal.

\section{Comparativos por grupo}

Para comparar las respuestas de los distintos grupos (por género, edad, semestre, rendimiento académico) en cada escala (epistémica y reproductiva) se utilizó la prueba t de Student para muestras independientes. En cuanto a los comparativos por género, se encontraron diferencias significativas en creencias de tipo epistémico. Las mujeres presentaron un mayor nivel que los varones $(\mathrm{t}=3.4 ; \mathrm{p}=.001)$, sin embargo, no se observaron diferencias significativas en las concepciones de tipo reproductivo entre las mujeres y los varones.

$\mathrm{Al}$ analizar las diferencias en concepciones de tipo epistémico y concepciones de tipo reproductivo respecto de la edad, no se encontraron diferencias significativas entre los grupos.

Respecto de los comparativos por semestre, únicamente se encontraron diferencias significativas en las concepciones de tipo reproductivo, pero no en las concepciones de tipo epistémico. El grupo que cursa $1^{\circ}-3^{\circ}$ presentó un mayor nivel medio en creencias reproductivas, en comparación con el que cursa $4^{\circ}-6^{\circ}(t=2.04 ; \mathrm{p}=$ .046). Sin embargo, no se observaron diferencias significativas entre los estudiantes que cursan $4^{\circ}-6^{\circ}$ y $7^{\circ}-10^{\circ}$, ni entre los que cursan $1^{\circ}-3^{\circ}$ y $7^{\circ}-10^{\circ}$.

En lo que concierne a los comparativos por rendimiento académico, se encontró que el grupo con promedio 9.0-10 presentó un nivel medio de creencias reproductivas, en comparación con el grupo con promedio 7.0-8.9 que obtuvo un nivel bajo $(\mathrm{t}=2.3 ; \mathrm{p}=.024)$, sin embargo, no se encontraron diferencias significativas en las creencias de tipo epistémico respecto al rendimiento académico. 


\section{DisCuSIÓN Y CONCLUSIONES}

Este estudio se centró en indagar las concepciones de escritura de estudiantes universitarios de medicina en sus dimensiones epistémica y reproductiva, además de valorar los tipos de concepciones de escritura en función de las variables sociodemográficas: género, edad, semestre y rendimiento académico.

Respecto a las concepciones de escritura en general, en consonancia con la literatura (Villalón y Mateos, 2009), hemos corroborado que los estudiantes no presentan una plena consciencia de creencias de tipo epistémico, prevaleciendo aún creencias de tipo reproductivo. Esto implica que si bien los jóvenes del estudio comparten una visión más elaborada sobre la escritura, como herramienta conceptual y retórica, también subyace la concepción de reproducir mediante la escritura la información que conocen y/o que aportan los autores (White y Bruning, 2005).

En cuanto a las concepciones de escritura en relación con el género, un dato destacable es que las mujeres mostraron mayor concordancia con las concepciones epistémicas, en relación con los varones. Sin embargo, mujeres y varones comparten un nivel medio de concordancia con las concepciones reproductivas. Los hallazgos nos llevan a inferir que las jóvenes de medicina todavía no presentan una clara idea del valor de la escritura como recurso para el aprendizaje, debido a que, de manera similar que los varones, aún conservan ideas reproductivas de nivel medio, lo cual podría influir de manera desfavorable en la calidad de los textos. Este resultado coincide con la investigación de Villalón, Mateos y Cuevas (2015) con estudiantes de bachillerato, en la que las jóvenes mostraron una mayor consciencia que los varones sobre el valor epistémico de la escritura, pero esta no se encontraba consolidada al conservar un nivel elevado de creencias reproductivas que a su vez coincidían con las del género masculino.

En lo referente a la edad, los estudiantes de distintas edades comparten un nivel elevado de concordancia respecto de las concepciones epistémicas de escritura. Sin embargo, los estudiantes de menor edad (17-19 años) manifestaron un nivel medio de concepciones reproductivas de escritura, a diferencia de los de mayor edad (2022 años y 23-27 años), quienes mostraron un nivel bajo de creencias reproductivas. Estos datos se asemejan a los resultados que se obtuvieron por semestre académico, pues mientras los estudiantes de $1^{\circ}-3^{\circ}$ presentaron un nivel medio de concordancia con las creencias reproductivas, los estudiantes de semestres más avanzados $\left(4^{\circ}-6^{\circ}\right.$, $\left.7^{\circ}-10^{\circ}\right)$, mostraron un bajo nivel de consistencia con estas concepciones.

Los presentes resultados, si bien no son significativos estadísticamente, lo cual coincide con investigaciones previas (Castells et al., 2015) en las que tampoco se observa una relación significativa entre el tipo de concepciones de escritura y los semestres de estudio universitario, sí nos marcan una tendencia en cuanto a la disminución de concepciones reproductivas mientras se avanza en el trayecto formativo. Por su parte, 
los datos obtenidos guardan similitud con el estudio de Villalón y Mateos (2009), en el cual los estudiantes de secundaria manifiestan mayor acuerdo con concepciones reproductivas que los universitarios. A su vez, estos hallazgos presentan coincidencia con distintos estudios (Hernández 2012, 2017; Hernández y Rodríguez, 2018; Villalón, 2010) en cuanto a que los estudiantes universitarios en general muestran concepciones transmisivas o reproductivas en menor grado que los de bachillerato. Sin embargo, que los universitarios no manifiesten un completo acuerdo con las concepciones epistémicas puede suponer una dificultad para la elaboración de ideas y de aprendizaje a partir de la escritura académica.

En el caso de los estudiantes con un nivel alto en su promedio de calificaciones (de 9 a 10) se observa mayor concordancia con las concepciones de tipo reproductivo que en aquellos de promedio menor (de 7 a 8). Este dato nos lleva a pensar que tener un mayor rendimiento académico no representa un cambio hacia una concepción más elaborada de la escritura. A su vez, dada la influencia de las prácticas de escritura académica en las concepciones de escritura y viceversa, esto podría significar la existencia de actividades de escritura, dentro de contextos educativos determinados, que pudiesen propiciar la reproducción de información, más que el desarrollo de nuevas ideas en torno a los contenidos sobre los cuales se escribe. Al respecto, habrá que considerar la influencia del contexto académico en las concepciones que los estudiantes desarrollan ya que, como plantean Hernández y Rodríguez (2018), la práctica de la escritura enfocada a la construcción de conocimiento puede no representar un objetivo para la formación en un tipo de disciplina particular.

Si bien son escasos los estudios sobre prácticas de escritura en el contexto de la enseñanza de la medicina, investigaciones realizadas en México (Hernández y Rodríguez, 2018) sobre distintas comunidades académicas (bachillerato, filosofía, historia, biología, psicología) reportan que en general la evaluación de los escritos académicos por parte de los docentes se centra en la ortografía y puntuación. Asimismo ponen de manifiesto que es habitual que las actividades de escritura de tipo complejo o aquellas que requieren un mayor nivel de integración y estructuración de contenidos, así como de reflexión por parte del estudiante, no suelen ser evaluadas en tanto piezas de expresión escrita sino en función del manejo de los contenidos aprendidos que demuestran.

Las cualidades de esta evaluación señalada por Hernández y Rodríguez (2018) es sabido que tienden a generar en los estudiantes ideas de tipo reproductivo sobre la escritura académica, en tanto la producción escrita se utiliza como medio para comunicar lo que otros ya han dicho y entre mejor se reproduzca este contenido se obtendrá una mejor evaluación. Por otra parte, los aspectos autorales o de toma de postura del alumno en este estudio fueron los menos valorados en todas las comunidades académicas analizadas, lo cual coincide con la investigación de Castelló et al. (2012) en universidades españolas. Estos aspectos que implican la expresión de lo 
que el estudiante piensa y la manera en que utiliza lo aprendido para reflexionar sobre nuevas ideas plausibles se asocian con las creencias de tipo transaccional (White y Bruning, 2005) o de tipo constructivo.

En general los resultados del estudio muestran que la metodología adoptada favoreció la identificación de las concepciones de escritura de los estudiantes. Ello coincide con trabajos previos que utilizan instrumentos estandarizados de autoinforme como una herramienta eficaz para acceder a las prácticas y creencias acerca de la escritura académica (Villalón y Mateos, 2009; Villalón, 2010). Por otro lado, somos conscientes de que para lograr una mayor comprensión de las concepciones de escritura de los estudiantes, en este caso de medicina, resulta necesario conocer las prácticas de escritura que llevan a cabo en su campo disciplinar, así como también la percepción que ellos tienen sobre estas en función de su utilidad para la construcción de nuevos aprendizajes o reproducción de contenidos. Asimismo, dada la naturaleza del presente estudio de tipo exploratorio, se realizó la indagación en una sola universidad, sin embargo, reconocemos que resulta necesario incluir en nuevos estudios a facultades de medicina de distintas universidades mexicanas, con la finalidad de lograr una mayor comprensión del fenómeno de la escritura en estudiantes de esta carrera.

Aunado a lo previo, en futuras investigaciones sería pertinente complementar el análisis de los resultados del cuestionario con la aplicación de otros instrumentos que permitan obtener información detallada sobre las prácticas de escritura que se desarrollan en el contexto de enseñanza y aprendizaje de la medicina, así también aplicar métodos como la entrevista con la finalidad de explorar la percepción que tienen los estudiantes sobre dichas prácticas en la disciplina que estudian.

\section{REFERENCIAS}

Bandura, A. (1982). Self-efficacy mechanism in human agency. American Psychologist, 37(2), 122-147. DOI: 10.1037/0003-066X.37.2.122.

Barbón, O., y Bascó, E. (2016). Clasificación de la actividad científica estudiantil en la educación médica superior. Educación Médica, 17(2), 55-60.

Bereiter, C., y Scardamalia, M. (1987). The psychology of written composition. Hillsdale, NJ: Erlbaum.

Bereiter, C. y Scardamalia, M. (1992). Dos modelos explicativos de los procesos de composición escrita. Infancia y Aprendizaje, 15(2), 43-64.

Boice, R. (1993). Writing blocks and tacit knowledge. The Journal of Higher Education, 64, 19-54. DOI: $10.2307 / 2959976$.

Boscolo, P., Arfé, B., y Quarisa, M. (2007). Improving the quality of students' academic writing: An intervention study. Studies in Higher Education, 32(4), 419-438.
Campbell, J., Smith, D., y Brooker, R. (1998). From conception to performance: How undergraduate students conceptualise and construct essays. Higher Education, 36(4), 449-469.

Castells, N., Mateos, M., Martín, E., Solé, I., y Miras, M. (2015). Profiles of self-perceived competencies and conceptions of academic writing in University students. Cultura y Educación, 27(3), 569-593.

Castelló, M., Iñesta, A., y Monereo, C. (2009). Towards selfregulated academic writing: An exploratory study with graduate students in a situated learning environment. Electronic Journal of Research in Educational Psychology, 7(3), 1107-1130.

Castelló, M., Mateos, M., Castells, N., Iñesta, A., Cuevas, I., y Sole, I. (2012). Academic writing practices in Spanish Universities. Electronic Journal of Research in Educational Psychology, 10(2), 569-590. 
Flower, L., Ackerman, J., y Peck, W. (1990). Reading to write: Exploring a cognitive and social process. Nueva York: Oxford University Press.

Hernández, G. (2012). Teorías implícitas de escritura en estudiantes pertenecientes a dos comunidades académicas distintas. Perfiles Educativos, 34(136), 42-62.

Hernández, G. (2015). Representaciones y prácticas de los estudiantes sobre escritura académica: diferencias entre distintas comunidades académicas. Memoria electrónica del XIII Congreso Nacional de Investigación Educativa, 16 al 20 de noviembre, Chihuahua, México.

Hernández, G. (2017). Las creencias de escritura en estudiantes de distintas comunidades académicas. En S. Espino y C. Barrón (coords.), La lectura y la escritura en la educación en México. Aproximaciones teóricas, experiencias aplicadas y perspectivas de futuro (pp. 61-83). Ciudad de México: IISUE-UNAM.

Hernández, G., y Rodríguez, E. (2018). Creencias y prácticas de escritura. Comparación entre distintas comunidades académicas. Revista Mexicana de Investigación Educativa, 23(79), 1093-1119.

Hounsell, D. (1984). Essay planning and essay writing. Higher Education Research and Development, 3(1), 13-31.

Lavelle, E. (1993). Development and validation of an inventory to assess processes in college composition. British Journal Educational Psychology, 63(3), 489-499.

Lavelle, E., y Bushrow, K. (2007). Writing approaches of graduate students. Educational Psychology, 27(6), 807-822.

Lavelle, E., y Zuercher, N. (2001). The writing approaches of university students. Higher Education, 42(3), 373-391.

Levin, T., y Wagner, T. (2006). In their own words: Understanding student conceptions of writing through their spontaneous metaphors in the science classroom. Instructional Science, 34(3), 227-278.

Lonka, K., Chow, A., Keskinen, J., Hakkarainen, K., Sandström, N., y Pyhältö, K. (2014). How to measure PhD students' conceptions of academic writing? Journal of Writing Research, 5(3), 245-269.

Martínez-Fernández, J. R., Corcelles, M., Bañales, G., Castelló, M., y Gutiérrez-Braojos, C. (2016). Exploring conceptions about writing and learning: undergraduates' patterns of beliefs and the quality of academic writing. Electronic Journal of Research in Educational Psychology, 14(1), 107-130. DOI: http://dx.doi.org/10.25115/ejrep.38.15045.

Märton, F., y Säljö, R. (1976). On qualitative differences in learning. Outcome and process. British Journal of Educational Psychology, 46(1), 4-11.

Miras, M. (2000). La escritura reflexiva. Aprender a escribir y aprender acerca de lo que se escribe. Infancia y aprendizaje, 89, 65-80.

Miras, M., Solé, I., y Castells, N. (2013). Creencias sobre lectura y escritura, producción de síntesis escritas y resultados de aprendizaje. Revista Mexicana de Investigación Educativa, 18, 437-459.

Nelson, J. (1990). This was an easy assignment: Examining how students interpret academic writing tasks. Technical report No. 43, Eric Information Analysis Products (IAP).

Olson, D. (1998). El mundo sobre el papel: el impacto de la lectura y escritura en la estructura del conocimiento. Barcelona: Gedisa.

Pozo, J. I., Scheuer, N., Mateos, M., y Pérez-Echeverría, M. (2006). Las teorías implícitas sobre el apr0endizaje y la enseñanza. En J. I. Pozo, N. Scheuer, M. del P. Pérez Echeverría, M. Mateos, E. Martín y M. De la Cruz (eds.), Nuevas formas de pensar la enseñanza y el aprendizaje. Las concepciones de profesores y alumnos (pp. 95-132). Barcelona: Graó.

Ríos-González, C. (2016). Escasa publicación científica en estudiantes de medicina de Paraguay. Carta al Director. Educación, 17(2), 80-81.

Villalón, R. (2010). Las concepciones de los estudiantes sobre la escritura académica [Tesis doctoral]. Recuperado de http:// www.tdx.cat/handle/10803/50700.

Villalón, R., y Mateos, M. (2009). Concepciones sobre la escritura académica: cómo conciben los estudiantes la escritura académica. Infancia y Aprendizaje, 32(2), 219- 232.

Villalón, R., Mateos, M., y Cuevas, I. (2015). High school boys and girls writing conceptions and writing self-efficacy beliefs: What is their role in writing performance? Educational Psychology, 35(6), 653-674.

White, M. J., y Bruning, R. (2005). Implicit writing beliefs and their relation to writing quality. Contemporary Educational Psychology, 30(2), 166-189.

Cómo citar este artículo:

Gaeta González, M. L., Zanotto González, M., y González-Ocampo, G. (2020). Concepciones de escritura académica en estudiantes de medicina. IE Revista de Investigación Educativa de la REDIECH, 11, e855. doi: 10.33010/ie_rie_rediech.v11i0.855. 